\title{
Theoretical Description of Carrier Mediated Magnetism in Cobalt Doped ZnO
}

\author{
Aron Walsh, Juarez L. F. Da Silva, and Su-Huai Wei \\ National Renewable Energy Laboratory, Golden, Colorado 80401, USA
}

(Received 4 March 2008; published 23 June 2008)

\begin{abstract}
Substitutional cobalt in $\mathrm{ZnO}$ has a weak preference for antiferromagnetic ordering. Stabilization of ferromagnetism is achieved through $n$-type doping, which can be understood through a band coupling model. However, the description of the transition to a ferromagnetic ground state varies within different levels of band theory; issues arise due to the density functional theory underestimation of the band gap of $\mathrm{ZnO}$, and the relative position of the nominally unfilled Co $t_{2 d}$ states. We examine these limitations, including approaches to overcome them, and explain the contradictions in previous studies, which drastically overestimate the doping threshold for magnetic ordering.
\end{abstract}

DOI: 10.1103/PhysRevLett.100.256401

The potential to simultaneously tune both charge and spin in solid state materials has lead to great interest in the field of spintronics [1]. The ultimate aim is a controllable room temperature semiconducting ferromagnet, which could be used in magnetoelectric and magnetotransport devices. Intrinsically magnetic semiconductors generally possess relatively low Curie temperatures $\left(T_{C}\right)$; however, it has been proposed that incorporating transition metal or rare earth ions into a nonmagnetic semiconductor host lattice, forming a dilute magnetic semiconductor (DMS), may help raise $T_{C}$ above room temperature [2,3].

In DMS materials, magnetism is influenced and controlled by the presence of charge carriers in the form of holes (GaAs:Mn) or electrons (GaN:Gd). There is still great debate over the fundamental mechanism behind the origin of the observed high temperature magnetic alignment in many of these systems. The situation is not helped by large variation in both experimental and theoretical studies [4-8].

Cobalt doped $\mathrm{ZnO}$ has become a focus of attention due to its reported high $T_{C}$ of up to $700 \mathrm{~K}$ and, most promisingly, the reversible cycling of FM ordering [9]. Coupled with existing optical and electrical properties of $\mathrm{ZnO}$, the addition of controllable magnetism could make it a technologically essential material. It has been reported that at Co concentrations of less than $10 \%$, the solubility is good and $\mathrm{Co}$ occupies a substitutional $\mathrm{Zn}$ site in $\mathrm{a}+2$ charge state $[9,10]$.

The stability of the $\mathrm{Co}$ (II) $3 d^{7} 4 s^{0}$ configuration in fourfold tetrahedral coordination sites is demonstrated by $\mathrm{Co}_{3} \mathrm{O}_{4}$ [11]. The roughly $T_{d}$ crystal field splits the Co $3 d$ states into lower $e_{d}$ and upper $t_{2 d}$ states. This results in the occupation of all the majority spin $3 d$ states and minority spin $e_{d}$ states, with a local Co magnetic moment close to 3 $\mu_{B}$. The splitting between filled $e_{d}$ and empty minority $t_{2 d}$ states for tetrahedral Co is typically on the order of $1.5-$ $2 \mathrm{eV}$ [12]. In a wide gap semiconductor such as $\mathrm{ZnO}\left(E_{g}=\right.$ $3.4 \mathrm{eV}$ ), this would place the minority spin Co $t_{2 d}$ states within the band gap, as has been reported from both optical and magnetic measurements for $\mathrm{ZnO}$ :Co [13-15]. Surpris-
PACS numbers: 71.20.Nr, 71.15.Mb, 75.10.-b, 75.50.Pp

ingly, this is not the case in previous theoretical studies [58].

From consideration of Co $3 d$ band coupling, FM interactions between occupied majority $t_{2 d}$ states results in no net energy gain due to filled bonding and antibonding combinations, while AFM interactions can result in a small energy gain (Fig. 1). Partial occupation of the empty minority $t_{2 d}$ states will result in a transition to FM ordering through the energy gained from the filling of the bonding state. Within such a band coupling model [16,17], the energy difference between the FM and AFM phases can be described as

$$
\Delta E_{\mathrm{FM}-\mathrm{AFM}}=-2 m_{e} \Delta_{d d}^{2}+2\left[\left(3-m_{e}\right) \Delta_{d d}^{1,2}\right],
$$

where $m_{e}$ is the additional number of electrons per Co(II) ion and $\Delta_{d d}^{2}$ and $\Delta_{d d}^{1,2}$ are the direct exchange and superexchange parameters, respectively (Fig. 1).
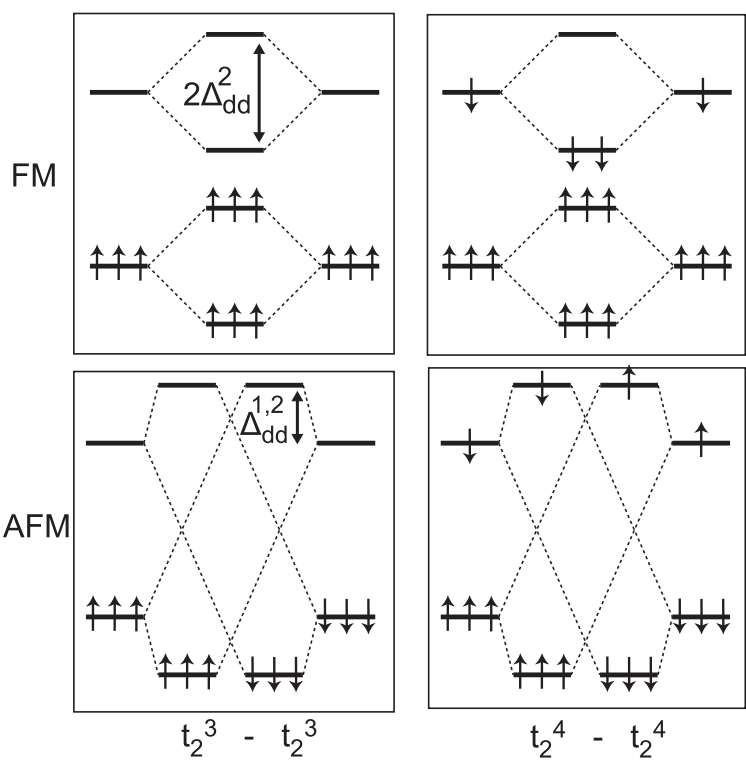

FIG. 1. Coupling of the $\mathrm{Co}_{2 d}$ levels for $\mathrm{Co}_{\mathrm{Zn}}$ substitution in $\mathrm{ZnO}$ for (left) the pure host system and (right) an $n$-type doped system. 
Coupling between the relatively localized Co $3 d$ states is possible through mediation of $\mathrm{O} p$ orbitals, which are of the same $t_{2}$ symmetry and can facilitate superexchange interactions. This is consistent with experiment, where reducing conditions, in the form of the addition of $\mathrm{Zn}$ vapor or low $\mathrm{O}_{2}$ partial pressure, are needed to stabilize FM ordering [9]. Extrinsic $n$-type doping (e.g., Al) has also been shown to have a similar effect [15]. In contrast, band structure studies of $\mathrm{ZnO}$ :Co have reported preference for both FM [5] and AFM [6] ordering, in addition to no preferred magnetic alignment [7].

In this work, we demonstrate that careful consideration of the electronic structure description is needed for accurate modeling of this system. In particular, the relative positions of the intrinsic $\mathrm{ZnO}$ conduction band and unfilled minority Co $t_{2 d}$ levels are shown to determine the magnetic behavior and hence control the onset of ferromagnetism with respect to carrier concentration.

Calculations were performed on a 72 atom wurtzite $\mathrm{ZnO}$ supercell using gradient corrected density functional theory [18-20] (PBE-DFT) as implemented in VASP [21]. A $2 \times 2 \times 2$ Monkhorst Pack $k$-point grid and a $500 \mathrm{eV}$ plane-wave cutoff were employed. To overcome errors associated with the DFT self-interaction, calculations were also performed using DFT corrected for on-site Coulombic interactions (DFT $+U_{d}$ ), and DFT with exact Fock exchange (hybrid-DFT). The latter were performed using a unreleased version of VASP [22]. For the DFT $+U_{d}$ calculations, the Co $3 d$ states were corrected by $U-J=$ $2 \mathrm{eV}$, which has been shown to result in Co $d$ - $d$ splitting consistent with experiment for $\mathrm{Co}_{3} \mathrm{O}_{4}$ [11], and $U-J=$ $5 \mathrm{eV}$ was applied to the $\mathrm{Zn} 3 d$ states. The $\mathrm{ZnO}$ band gaps at the DFT, DFT $+U_{d}$ and hybrid-DFT levels are $0.8 \mathrm{eV}$, $1.4 \mathrm{eV}$ and $3.4 \mathrm{eV}$ (PBE0), respectively. For bulk $\mathrm{ZnO}$, the cell size and atomic coordinates were fully relaxed. For Co containing systems, the lattice constant was fixed, but the internal positions were allowed to relax. Carriers were introduced by partially filling the lowest unoccupied bands, with a uniform background charge employed to ensure that the net charge of the periodic cell remains zero.

For nearest neighbor $\mathrm{Co}_{\mathrm{Zn}}$ pairs, the calculated ground state magnetic ordering at both the DFT and DFT $+U_{d}$ levels shows enhanced AFM stabilization energies of 37 and $31 \mathrm{meV}$ per Co pair, respectively. This is in agreement with the absence of ordered magnetism at room temperature, as the local moments will randomly orientate under the influence of thermal fluctuations.

Experimentally, reducing conditions are needed to stabilize FM ordering. Partial filling of the nominally empty minority spin Co $t_{2 d}$ states is expected to result in a transition to FM ordering (Fig. 1). We have simulated the effect of donors by calculating both FM and AFM ordering of nearest neighbor Co pairs as a function of electrons. This approximates that the Co ions are more directly affected by the addition of charge carriers than the structural effects of the underlying donor defects.
Surprisingly, for DFT, the addition of up to one electron per Co does not significantly alter the energetic difference between AFM and FM ordering, $\Delta E_{\mathrm{FM}-\mathrm{AFM}}$ (Fig. 2). This also holds true for DFT $+U_{d}$. This effect originates from errors in the relative band level positions as summarized in Fig. 3. Absorption measurements indicate that the occupied minority $e_{d}$ states lie approximately $1.5 \mathrm{eV}$ above the $\mathrm{ZnO}$ valence band maximum (VBM) [13]. At the DFT level, this separation is reduced to $0.8 \mathrm{eV}$, and as this coincides with the underestimated conduction band minimum (CBM) of $\mathrm{ZnO}$, there is partial occupation of both the $\mathrm{ZnO} C B M$ and the Co $e_{d}$ states producing an incorrect metallic electronic structure. In this case, adding extra electrons to the unoccupied $e_{d}$ state reduces the magnetic moment and the exchange splitting between the majority and minority spin states, which slightly enhances AFM ordering through the increase of $\Delta_{d d}^{1,2}$ [Eq. (1)].

DFT $+U_{d}$ helps by partially correcting the band gap underestimation, but fails to resolve the conflict with the experimental electronic structure: while the VBM-Co $e_{d}$ separation is good, and the Co minority $e_{d}-t_{2 d}$ splitting of $1.9 \mathrm{eV}$ is in agreement with available data, the $\mathrm{ZnO}$ band gap is still too small, such that the unoccupied $t_{2 d}$ states lie well above the calculated CBM. This results in the occupation of $\mathrm{Zn} 4 s$ bands before Co $t_{2 d}$, and hence a high concentration of carriers are required to stabilize FM ordering. Once the initial threshold is overcome, FM coupling between the Co ions becomes rapidly stabilized as expected from our initial band coupling considerations. The addition of 1.5 electrons per Co results in a $\Delta E_{\mathrm{FM}-\mathrm{AFM}}$ of $120 \mathrm{meV}(291 \mathrm{meV})$ for DFT $\left(\mathrm{DFT}+U_{d}\right)$; however, such high doping levels corresponding to $4 \times$ $10^{21} \mathrm{~cm}^{-3}$ are physically unrealistic for the initial onset of ferromagnetism (Fig. 2).

Another widely used approach to compensate for the DFT band gap underestimation is hybrid-DFT, which con-

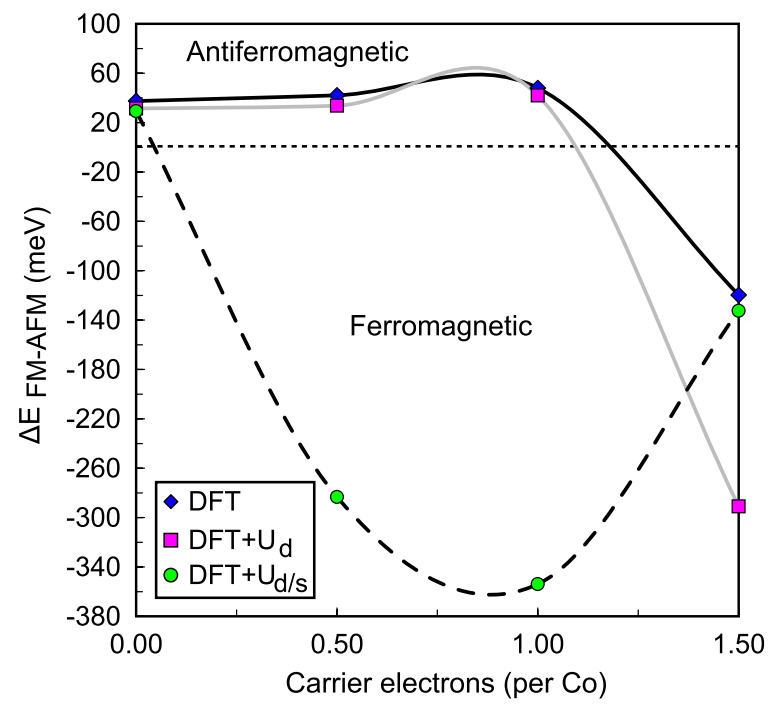

FIG. 2 (color online). Calculated magnetic ordering energies in $\mathrm{ZnO}: \mathrm{Co}$ as a function of carrier electrons. 


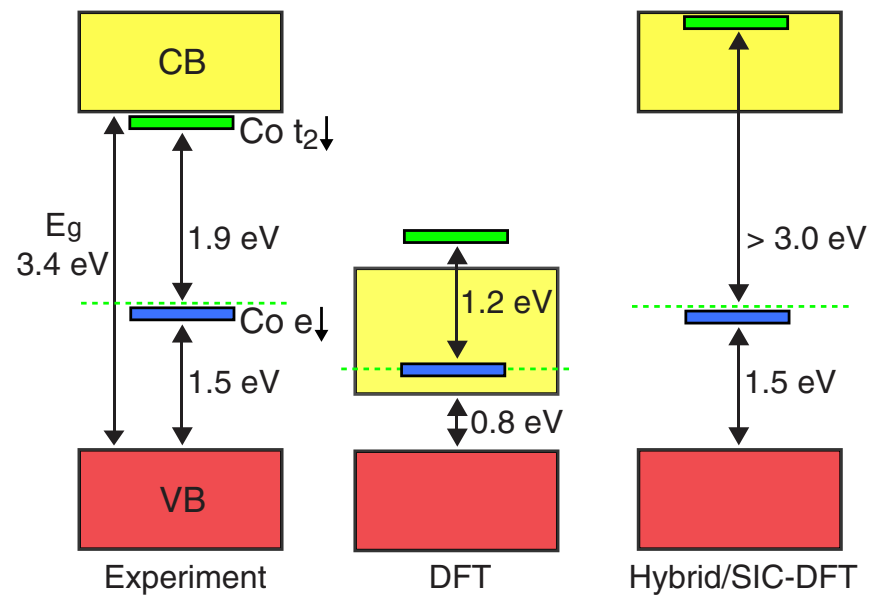

FIG. 3 (color online). Schematic band level diagram comparing the minority $\mathrm{Co} d$ levels in $\mathrm{ZnO}$ derived from experiment [13] and DFT calculations. The highest occupied state is indicated by the dashed horizontal line.

tains the proportion of exact nonlocal Fock exchange as an adjustable parameter; i.e., the exchange-correlation functional becomes

$$
E_{X C}^{\mathrm{Hybrid}}=(1-x) E_{X}^{\mathrm{PBE}}+x E_{X}^{\mathrm{Fock}}+E_{C}^{\mathrm{PBE}} .
$$

Indeed the B3LYP functional has been applied to treat $\mathrm{ZnO}: \mathrm{Co}$, but again unphysically high levels of electron doping are needed to stabilize FM ordering [6]. From our analysis, this originates from a strong system dependence of the exact exchange, which cannot be overcome within this methodology. For example, to reproduce the experimental band gap of $\mathrm{ZnO}, 25 \%$ of Fock exchange is required, while for $\mathrm{Co}_{3} \mathrm{O}_{4}$ less than $5 \%$ exchange is adequate (Fig. 4). This is due to the localized nature of the $\mathrm{Co}_{3} \mathrm{O}_{4}$ band edges which contain a stronger self-interaction in comparison to $\mathrm{ZnO}$ whose $\mathrm{CBM}$ is a delocalized cationanion $s$ state. At the high levels of exchange needed to treat the band gap of $\mathrm{ZnO}$, the Co $d$ - $d$ splittings are overestimated in the order of $300 \%$. Recently, self-interactioncorrected (SIC) DFT has also been applied to ZnO:Co, but to similar avail, with the Co $t_{2 d}$ levels situated well above the $\mathrm{ZnO}$ CBM $[8,23]$. In all such approaches, the CBM states are preferentially filled and act as a strong barrier to ferromagnetism.

An alternative solution can be found through application of an orbital dependent potential to control the band gap of $\mathrm{ZnO}$ and the Co $d-d$ splitting independently. This could be done by either adding a Christensen potential [24,25] or by applying a Coulomb $U$ on both the $s$ and $d$ orbitals to further raise the CBM level [26,27]. Through the application of DFT $+U_{d / s}$ we can obtain an electronic structure description consistent with experiment; however, other approaches can also be used to correct the DFT band errors, and the magnetic physical picture discussed in this paper is not affected by the method used. As shown in the calculated density of states (Fig. 5), at high binding

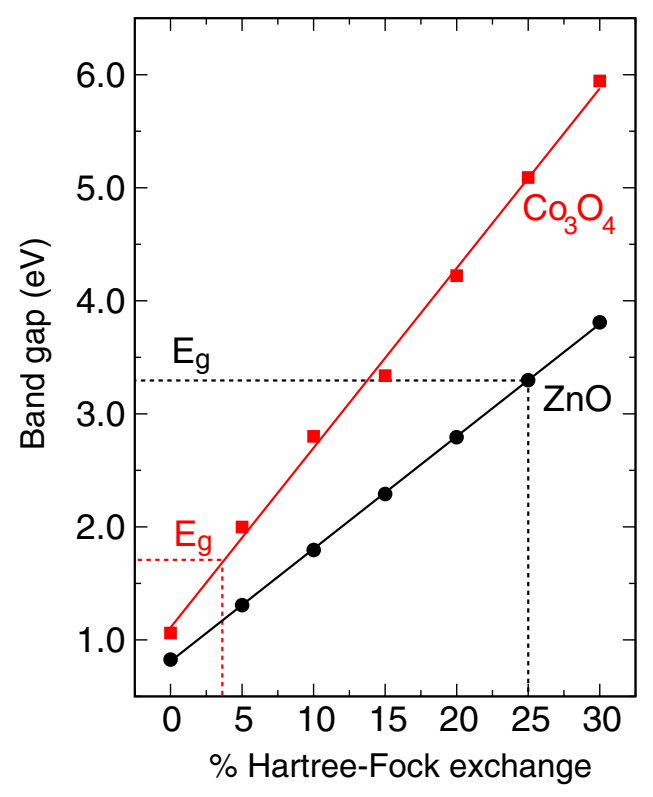

FIG. 4 (color online). Calculated $\Gamma-\Gamma$ valence and conduction band separations in wurtzite $\mathrm{ZnO}$ and spinel $\mathrm{Co}_{3} \mathrm{O}_{4}$ as a function of Fock exchange in hybrid-DFT. The corresponding experimental band gaps are indicated by dashed lines [30].

energy are the $\mathrm{Zn} 3 d$ states $(-9 \mathrm{eV})$, which are followed by the main $\mathrm{O} p$ valence band. The topmost occupied states are the minority Co $e_{d}$ states, with the unoccupied $t_{2 d}$ states present around the onset of the $\mathrm{ZnO}$ conduction band. This more realistic description radically lowers the threshold for the onset of ferromagnetism. On electron doping, even the addition of 0.5 electrons to the system results in a strong stabilization of FM ordering with $\Delta E_{\mathrm{FM}-\mathrm{AFM}}=$ $-283 \mathrm{meV}$ (Fig. 2). From our calculations, a maximum in the FM interaction is reached at the addition of about one electron per Co ion $(354 \mathrm{meV})$, corresponding to a carrier concentration of $2 \times 10^{21} \mathrm{~cm}^{-3}$. This maximum is somewhat lower than the 1.5 electrons predicted from the ideal band coupling model [Eq. (1)], where the minority spin bonding combination would be fully occupied (Fig. 1). The difference is due to the effects of partially filling of the $\mathrm{ZnO} \mathrm{CBM}$ and the enhancement of the superexchange $\Delta_{d d}^{1,2}$ term in Eq. (1) at higher carrier concentrations.

From our results we can clearly state that the electronic structure of $\mathrm{ZnO}: \mathrm{Co}$ is consistent with carrier mediated ferromagnetism. Both the band coupling interpretation and the calculated transition to FM ordering, on the addition of a small number of carriers, indicate more general magnetic interactions in contrast to hypothesized defect complexes $[8,28]$. While our calculations do not rule out the existence or contributions of such mechanisms, they do show that the transition to FM ordering should be independent of the nature of the carriers.

We have demonstrated that the fundamental magnetic interactions in Co doped $\mathrm{ZnO}$ can be understood from band coupling of the Co $3 d$ states. The system is intrinsically AFM, but the addition of charge carriers (electrons) will 


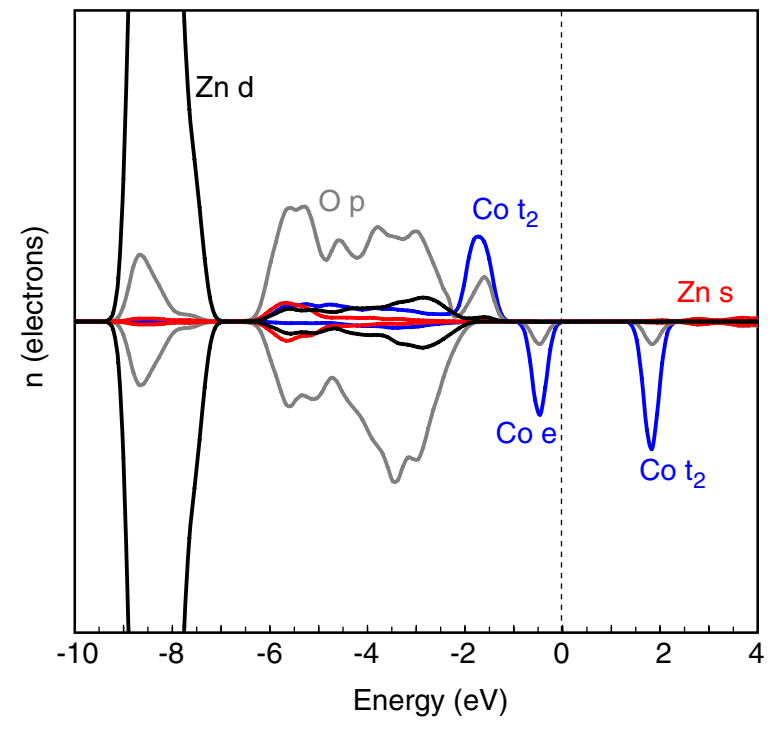

FIG. 5 (color online). $\quad$ DFT $+U_{d / s}$ calculated density of states for $\mathrm{Zn}_{34} \mathrm{O}_{36}: \mathrm{Co}_{2}$ in an antiferromagnetic configuration. The Co states are $\times 2$ for clarity and the Fermi level is indicated by the dashed vertical line.

result in a transition to FM ordering through enhanced coupling between the Co $t_{2 d}$ states. Within DFT, the ground state of weak AFM behavior is correctly described, but the transition to FM ordering is hampered by the severe band gap underestimation for $\mathrm{ZnO}$. The onset of ferromagnetism is incorrectly described due to the preferential occupation of the $\mathrm{ZnO}$ conduction band before unoccupied minority Co $3 d$ bands. This failure cannot be overcome within the realms of hybrid-DFT, due to the sensitive dependence of Co on the addition of exact Fock exchange, in comparison to $\mathrm{Zn}$ : for all values of exchange, the Co minority $e_{d}-t_{2 d}$ splitting is larger than the $\mathrm{ZnO}$ band gap. Through the application of an orbital dependent potential $\left(\mathrm{DFT}+U_{d / s}\right)$, a reasonable description of the band structure and level occupations can be obtained, which reveals the correct origins of ferromagnetic phase stabilization in this system.

Improvement in the treatment of exchange-correlation effects in DFT is required to accurately treat magnetic doping of semiconductors within a fully ab initio framework, i.e., without the introduction of multiple system dependent parameters. Higher-order methods such as self-consistent GW theory [29] and dynamical mean field theory (DMFT) with total energy may offer a solution in the future. We expect that electron mediated DMS systems with calculated band gaps smaller than the typical transition metal $d-d$ splittings will be prone to these DFT band filling errors, including $\mathrm{GaN}, \mathrm{In}_{2} \mathrm{O}_{3}$ and $\mathrm{SnO}_{2}$. The limitations of the method adopted should be carefully considered in future theoretical studies.
We thank G. Kresse for the provision of VASP 5.1. The work is supported by the U.S. Department of Energy (Contract No. DE-AC36-99GO10337).

[1] D. D. Awschalom and M. E. Flatte, Nature Phys. 3, 153 (2007).

[2] T. Dietl et al., Science 287, 1019 (2000).

[3] K. Sato and H. Katayama-Yoshida, Semicond. Sci. Technol. 17, 367 (2002).

[4] R. Janisch, P. Gopal, and N. A. Spaldin, J. Phys. Condens. Matter 17, R657 (2005).

[5] P. Gopal and N. A. Spaldin, Phys. Rev. B 74, 094418 (2006).

[6] E.-J. Kan et al., J. Appl. Phys. 102, 033915 (2007).

[7] C. H. Patterson, Phys. Rev. B 74, 144432 (2006).

[8] C. D. Pemmaraju et al., arXiv:0801.4945v1.

[9] K. R. Kittilstved et al., Phys. Rev. Lett. 97, 037203 (2006).

[10] Y.Z. Liu et al., Appl. Phys. Lett. 90, 154101 (2007).

[11] A. Walsh et al., Phys. Rev. B 76, 165119 (2007).

[12] H. A. Weakliem, J. Chem. Phys. 36, 2117 (1962).

[13] K. R. Kittilstved, W. K. Liu, and D. R. Gamelin, Nat. Mater. 5, 291 (2006).

[14] A. Barla et al., Phys. Rev. B 76, 125201 (2007).

[15] A. J. Behan et al., Phys. Rev. Lett. 100, 047206 (2008).

[16] G. M. Dalpian and S.-H. Wei, Phys. Stat. Sol. B 243, 2170 (2006).

[17] G. M. Dalpian et al., Solid State Commun. 138, 353 (2006).

[18] W. Kohn and L. J. Sham, Phys. Rev. 140, A1133 (1965).

[19] P. Hohenberg and W. Kohn, Phys. Rev. 136, B864 (1964).

[20] J. P. Perdew, K. Burke, and M. Ernzerhof, Phys. Rev. Lett. 77, 3865 (1996).

[21] G. Kresse and J. Furthmüller, Phys. Rev. B 54, 11169 (1996).

[22] J. Paier et al., J. Chem. Phys. 124, 154709 (2006).

[23] M. Toyoda et al., J. Phys. B 376-377, 647 (2006).

[24] N. E. Christensen, Phys. Rev. B 30, 5753 (1984).

[25] S.-H. Wei and A. Zunger, Phys. Rev. B 48, 6111 (1993).

[26] H. Raebiger, S. Lany, and A. Zunger, Phys. Rev. B 76, 045209 (2007).

[27] Here we apply DFT $+U_{d}$ with an additional repulsive potential $\left(-U_{s}=56 \mathrm{eV}\right)$ on $\mathrm{O} s$. The delocalized $\mathrm{O} s$ states lie to high binding energy and are mildly effected by the application of $U$ (shifting by $\sim 3 \mathrm{eV}$ ). This has a weak effect of raising the $\mathrm{ZnO} \mathrm{CBM}$ state $(40 \% \mathrm{O} s)$. See EPAPS Document No. E-PRLTAO-101-028852 for further discussion and background on the band gap correction method adopted. For more information on EPAPS, see http://www.aip.org/pubservs/epaps.html..

[28] M. Venkatesan et al., Phys. Rev. Lett. 93, 177206 (2004).

[29] M. van Schilfgaarde et al., Phys. Rev. Lett. 96, 226402 (2006).

[30] The $\mathrm{Co}_{3} \mathrm{O}_{4}$ fundamental gap is found at $X(\sim 1.2 \mathrm{eV})$, corresponding to a $\Gamma$ separation of $1.7 \mathrm{eV}$ (Ref. [11]). 